\title{
The influence of chronic renal failure on the spectrum and antimicrobial susceptibility of uropathogens in community-acquired acute pyelonephritis presenting as a positive urine culture
}

Yeon Soon Jung, Ho Sik Shin ${ }^{*}$ and Hark Rim

\begin{abstract}
Background: The role of chronic renal failure (CRF) in the antimicrobial resistance of uropathogens in patients with community-acquired acute pyelonephritis (APN) remains poorly understood.

Method: We performed a retrospective analysis of 502 adults (54 men, 448 women; mean age $61.7 \pm 16.0$ years, range 18-98 years) who were treated for community-acquired APN at Kosin University Gospel Hospital (Busan, Republic of Korea) during a ten-year period (January 2000 to December 2009). We evaluated the spectra and antimicrobial susceptibility profiles of uropathogens in CRF and non-CRF patients with community-acquired APN that presented as a positive urine culture.

Results: The 502 adult subjects were classified as either non-CRF APN patients (336 patients, 66.9\%) or CRF APN patients (166 patients, $33.1 \%$ ) according to their estimated glomerular filtration rate. No significant differences in the sensitivity of $E$. coli to a third cephalosporin, aminoglycoside (except gentamycin), or ciprofloxacin were observed between non-CRF and CRF patients.

Conclusions: In our series of patients with community-acquired APN that initially presented as a positive urine culture, CRF did not influence the isolation rates of different uropathogens or their patterns of susceptibility to antimicrobials.
\end{abstract}

Keywords: Pyelonephritis Chronic Renal Failure, Antimicrobial Susceptibility

\section{Background}

Acute pyelonephritis (APN), an infectious disease of the renal parenchyma and pelvic region, is a significant and frequent cause of morbidity, resulting in more than 100,000 hospital admissions per year in the United States [1,2]. The most common pathogens in APN belong to the Enterobacteriaceae family, and Escherichia coli is the causative pathogen in more than $80 \%$ of cases [1]. Other microbes contributing to the pathogenesis of APN include Proteus spp, Klebsiella spp, and enterococci [3]. Risk factors that predispose women to APN

\footnotetext{
* Correspondence: danieljoseph@hanmail.net

Department of Internal Medicine, Kosin University College of Medicine, Busan, Republic of Korea
}

include diabetes mellitus (DM), incontinence, patient and family history of urinary tract infections (UTIs), and certain sexual behaviors [4]. Although DM is a risk factor, it was previously reported that DM does not influence the isolation rates of different uropathogens or their patterns of susceptibility to antimicrobials [5].

In general, CRF patients are known to be vulnerable to infection due to weakened immunity [6]. But, few data are available on the role of CRF as a risk factor for the development of antimicrobial resistance of the uropathogens [7]. For this reason, we have undertaken a study to evaluate the spectrum of etiologic agents and their profiles of antimicrobial resistance in a large series 
of non-CRF and CRF patients with community-acquired APN that presented as a positive urine culture.

\section{Methods \\ Patients}

We performed a retrospective analysis of the medical records of Korean adults who were admitted to Kosin University Gospel Hospital (Busan, Republic of Korea) due to community-acquired APN between January 2000 and December 2009 and evaluated the spectra and antimicrobial susceptibility profiles of uropathogens in both non-CRF and CRF patients with community-acquired APN that presented as a positive urine culture. The analysis included 502 adults aged 18 years or older (54 men, 448 women; mean age $61.7 \pm 16.0$ years, range $18-98$ years).

\section{Inclusion and Exclusion Criteria}

Our clinical diagnosis of APN was based on the study of Safrin et al. (1988) [8]. In addition to the clinical diagnosis, which was based on signs and symptoms of APN in adult patients, patients were required to meet at least one of the following three criteria: 1) a positive dipstick test for leukocyte esterase; 2) a white blood cell (WBC) count $\geq 5$ cells per high-power field microscopy on examination of centrifuged urine sediment; or 3) a WBC count $\geq 10$ cells/ $\mu \mathrm{L}$ in non-centrifuged urine.

The exclusion criteria were as follows: 1) less than 18 years of age; 2) history of chronic infection; 3 ) evidence of other infection; 4) previous administration (in the previous six months) of antibiotics; 5) urologic abnormalities; 6) hospital-acquired APN (infection acquired during hospital care which was not present or incubating at time of admission. Infections occurring more than 48 hours after admission are usually considered nosocomial) [9]; 7) presence of an indwelling bladder catheter; 8) anticancer therapy or immunosuppressive medication, such as steroids; 9) pregnancy; and 10) negative results on urine culture.

\section{Clinical and biochemical assessment}

Type 2 diabetes was diagnosed according to the Report of the Expert Committee on the Diagnosis and Classification of Diabetes Mellitus [10].

As a surrogate for renal function, we estimated the glomerular filtration rate (GFR) using a simplified form of the Modification of Renal Disease (MDRD) equation [11]:

Estimated GFR $(\mathrm{eGFR})=186 \times[\mathrm{Scr}]^{-1.154} \times\left[\mathrm{Age}^{-0.203} \times[0.742\right.$ if patient is female $]$

CRF was defined as follows [12]: eGFR less than 60 $\mathrm{mL} / \mathrm{min} / 1.73 \mathrm{~m}^{2}$ without kidney damage for more than three months.
Baseline urine specimens were collected using a sterile, midstream, clean-catch technique. Specimens were sent to a central laboratory for urinalysis and susceptibility testing, and a urine culture was performed on each sample. Central laboratories were required to use the methodology of the Clinical and Laboratory Standards Institute (CLSI) [13] with quality-control methods using appropriate American Type Culture Collection organisms. Quantitative urine culture was performed using a dipslide method; urine was also streaked onto MacConkey agar. After incubation at $37^{\circ}$ $\mathrm{C}$ for $24 \mathrm{~h}$, the microorganisms were identified using standard biochemical tests. Presence of a pathogen in the urine culture was confirmed based on a colony count of greater than or equal to $10^{5} \mathrm{CFU} / \mathrm{mL}$. In vitro susceptibility to antibiotics was performed using an agar diffusion method (Kirby Bauer) employing dried filter paper discs impregnated with specific concentrations of antimicrobial agents, in accordance with the National Committee for the Clinical Laboratory Standards [14]. Susceptibility testing results for pathogens present upon admission were categorized as susceptible or resistant, and susceptibility guidelines were taken from the CLSI guidelines [13]. We also investigated antibiotic sensitivities to ampicillin, cephalothin, cefuroxime, cefotaxime, cefazolin, ceftazidime, imipenem, gentamycin, amikacin, tobramycin, ciprofloxacin, and trimethoprim-sulfamethoxazole (TMP-SMX)

\section{Statistical analysis}

The results are presented as the mean \pm SD. We used Student's $t$ test to compare the means between groups. Differences in antibiotic sensitivity and uropathogen profiles between groups were analyzed using the $X^{2}$ test. The results were considered significant when the $P$ value was less than 0.05 . All statistical analyses were performed using the Statistical Package for the Social Sciences (SPSS), version 12.0 (SPSS Inc, Chicago, IL, USA).

\section{Results}

The characteristics of the 502 subjects enrolled in this study are shown in Table 1 . The mean age was $61.7( \pm$ 16.0 ) years, with $89.2 \%$ of the subjects being women and $20.1 \%$ being diabetic patients.

The etiologic microorganisms of APN are shown in Table 2. The most common cause of APN was E. coli (58.3\%, 293 cases), followed by K. pneumonia (12.7\%, 64 cases), Pseudomonas (4.1\%, 21 cases), and Enterococcus $(2.9 \%, 15$ cases $)$.

The antimicrobial sensitivity rates for E. coli are shown in Table 3 . The sensitivity rates were $100 \%$ for imipenem, 99.2\% for amikacin, 90.8\% for tobramycin, over $80 \%$ for third- and fourth-generation cephalosporin 
Table 1 Age-gender standardized baseline demographics and laboratory results according to eGFR

\begin{tabular}{|c|c|c|c|}
\hline eGFR & $\begin{array}{l}\geq 60 \mathrm{~mL} / \mathrm{min} / \\
1.73 \mathrm{~m}^{2} \\
\mathrm{n}=336\end{array}$ & $\begin{array}{l}<60 \mathrm{~mL} / \mathrm{min} / \\
1.73 \mathrm{~m}^{2} \\
\mathrm{n}=166\end{array}$ & $\begin{array}{l}p- \\
\text { value }\end{array}$ \\
\hline \multicolumn{4}{|l|}{ Characteristics } \\
\hline Age, years & $59.0 \pm 16.8$ & $67.3 \pm 12.4$ & 0.0001 \\
\hline Genders, \% & & & 0.168 \\
\hline Males & 12.2 & 7.8 & \\
\hline Females & 87.8 & 92.2 & \\
\hline Diabetes, \% & 20.8 & 21.4 & 0.906 \\
\hline \multicolumn{4}{|l|}{ Measurements } \\
\hline WBC, $\mathrm{mm}^{3}$ & $9,944 \pm 5,630$ & $11,882 \pm 6,233$ & 0.001 \\
\hline $\mathrm{Hb}, \mathrm{g} / \mathrm{dL}$ & $11.5 \pm 1.9$ & $11.1 \pm 1.9$ & 0.083 \\
\hline Platelet, mm $\mathrm{mm}^{3}$ & $\begin{array}{l}226,232 \pm \\
106,223\end{array}$ & $\begin{array}{l}212,380 \pm \\
112,614\end{array}$ & 0.182 \\
\hline BUN, IU/L & $12.4 \pm 6.0$ & $27.4 \pm 14.4$ & 0.0001 \\
\hline Creatinine, IU/L & $0.72 \pm 0.15$ & $1.62 \pm 0.64$ & 0.0001 \\
\hline eGFR, $\mathrm{mL} / \mathrm{min} / 1.73 \mathrm{~m}^{2}$ & $98 \pm 32$ & $39 \pm 13$ & 0.0001 \\
\hline Sodium, mEq/L & $136 \pm 5$ & $135 \pm 6$ & 0.157 \\
\hline Potassium, mEq/L & $3.8 \pm 0.5$ & $4.0 \pm 0.7$ & 0.003 \\
\hline Protein, g/dL & $6.6 \pm 3.6$ & $6.3 \pm 0.9$ & 0.334 \\
\hline Albumin, $\mathrm{g} / \mathrm{dL}$ & $3.4 \pm 0.6$ & $3.2 \pm 0.6$ & 0.002 \\
\hline Total Bilirubin, mg/dL & $1.36 \pm 2.10$ & $1.65 \pm 2.14$ & 0.172 \\
\hline Direct Bilirubin, mg/dL & $0.74 \pm 1.61$ & $0.93 \pm 1.50$ & 0.233 \\
\hline AST, IU/L & $47.2 \pm 76.9$ & $72.4 \pm 249.3$ & 0.216 \\
\hline$A L T, I U / L$ & $39.0 \pm 71.6$ & $37.2 \pm 87.2$ & 0.813 \\
\hline ALP, IU/L & $130.5 \pm 130.3$ & $124.7 \pm 133.6$ & 0.675 \\
\hline$r-G T P, I U / L$ & $110.3 \pm 202.9$ & $82.9 \pm 115.2$ & 0.085 \\
\hline PT, second & $92.5 \pm 21.4$ & $84.2 \pm 22.5$ & 0.001 \\
\hline PT INR & $1.09 \pm 0.29$ & $1.19 \pm 0.65$ & 0.048 \\
\hline $\mathrm{PTT}$, second & $37.8 \pm 13.5$ & $39.8 \pm 10.9$ & 0.133 \\
\hline $\mathrm{CRP}, \mathrm{mg} / \mathrm{dL}$ & $6.6 \pm 7.4$ & $8.8 \pm 8.5$ & 0.045 \\
\hline HS-CRP, mg/dL & $7.0 \pm 7.8$ & $11.0 \pm 9.4$ & 0.002 \\
\hline $\begin{array}{l}\text { Total cholesterol, mg/ } \\
\text { dL }\end{array}$ & $163 \pm 47$ & $154 \pm 49$ & 0.180 \\
\hline $\begin{array}{l}\text { HDL cholesterol, mg/ } \\
\mathrm{dL}\end{array}$ & $41 \pm 16$ & $36 \pm 17$ & 0.032 \\
\hline $\begin{array}{l}\text { LDL Cholesterol, mg/ } \\
\mathrm{dL}\end{array}$ & $102 \pm 40$ & $85 \pm 38$ & 0.008 \\
\hline Triglycerides, mg/dL & $105 \pm 60$ & $131 \pm 107$ & 0.041 \\
\hline Chloride, mEq/L & $100 \pm 5$ & $101 \pm 7$ & 0.575 \\
\hline total $\mathrm{CO}_{2}, \mathrm{mEq} / \mathrm{L}$ & $26.7 \pm 4.8$ & $22.7 \pm 5.6$ & 0.0001 \\
\hline ESR, mm/hour & $30.5 \pm 23.7$ & $36.2 \pm 23.0$ & 0.030 \\
\hline Urine RBC (0-4+) & $1.32 \pm 2.22$ & $1.36 \pm 1.91$ & 0.003 \\
\hline Urine protein $(0-4+)$ & $0.65 \pm 0.89$ & $0.83 \pm 1.00$ & 0.0001 \\
\hline Urine WBC (0-4+) & $2.29 \pm 2.62$ & $2.99 \pm 2.76$ & 0.0001 \\
\hline
\end{tabular}

antibiotics, $71.7 \%$ for ciprofloxacin, and $61.4 \%$ for TMPSMX. However, the sensitivity rates for gentamycin and ampicillin were low (42.9\% and $39.4 \%$, respectively).

Age-gender standardized baseline demographics and laboratory results according to eGFR are shown in Table 1 . The rates of diabetes in the non-CRF and CRF
Table 2 Etiologic Microorganisms of APN

\begin{tabular}{llll}
\hline Pathogen & Male & Female & Total \\
\hline E.coli & 12 & 281 & 293 \\
K.pneumoniae & 8 & 56 & 64 \\
Proteus & 3 & 11 & 14 \\
Pseudomonas & 9 & 12 & 21 \\
Enterococcus & 2 & 13 & 15 \\
G(+) & 16 & 50 & 66 \\
G(-) & 4 & 25 & 29 \\
Total & 54 & 448 & 502 \\
\hline
\end{tabular}

APN: Acute Pyelonephritis, $\mathrm{G}(+)$ : Gram positive microorganism

$\mathrm{G}(-)$ : Gram negative microorganism

groups were $20.8 \%$ and $21.4 \%$, respectively ( $p=N S)$. The mean ages were 59.0 ( \pm 16.8 ) years in the non-CRF group and $67.3( \pm 12.4)$ years in the CRF group $(\mathrm{p}=$ $0.0001)$, and the values of HS-CRP were $7.0( \pm 7.8) \mathrm{mg} /$ $\mathrm{dL}$ in the non-CRF group and $11.0( \pm 9.4) \mathrm{mg} / \mathrm{dL}$ in the CRF group $(\mathrm{p}=0.002)$. The values for WBC $(\mathrm{p}=0.001)$ and ESR ( $p=0.03$ ) were higher in the CRF group than in the non-CRF group.

The antimicrobial sensitivity rates (\%) for E. coli according to eGFR are shown in Table 3. The antimicrobial sensitivities to ampicillin in the non-CRF group and CRF group were $37.8 \%$ and $20.0 \%$, respectively $(\mathrm{p}=0.024)$, and sensitivities to gentamycin in the non-CRF group and CRF group were $71.9 \%$ and $51.9 \%(\mathrm{p}=0.016)$, respectively. Significantly higher resistance of $E$. coli to amoxicillin and gentamycin was noted in isolates recovered in patients with CRF. No significant differences in the sensitivity of $E$. coli were observed between non-CRF and CRF patients.

The isolation rates (\%) of uropathogens in APN patients with or without CRF are shown in Table 4. The

Table 3 Antimicrobial Sensitivity Rate (\%) for E.coli according to eGFR

\begin{tabular}{llll}
\hline Antibiotics & $\begin{array}{l}\geq 60 \mathrm{~mL} / \\
\mathbf{m i n} / \\
\mathbf{1 . 7 3} \mathbf{~ m}^{2}\end{array}$ & $\begin{array}{l}<60 \mathrm{~mL} / \\
\mathbf{m i n} /\end{array}$ & $\begin{array}{l}\mathrm{p} \text { - } \\
\text { value }\end{array}$ \\
\hline Ampicillin & 37.8 & 20.0 & \\
Cephalothin & 45.5 & 36.0 & 0.024 \\
Cefuroxime & 76.9 & 69.4 & 0.428 \\
Cefotaxime & 82.0 & 76.5 & 0.413 \\
Cefozolin & 66.7 & 47.1 & 0.162 \\
Ceftazidime & 83.2 & 75.5 & 0.259 \\
Cepefime & 83.6 & 80.4 & 0.613 \\
Imipenem & 95.5 & 100 & 0.126 \\
Gentamycin & 71.9 & 51.9 & 0.016 \\
Amikacin & 96.3 & 90.0 & 0.112 \\
Tobramycin & 87.0 & 82.0 & 0.404 \\
Ciprofloxacin & 72.7 & 66.0 & 0.389 \\
Trimethoprime- & 57.9 & 56.9 & 0.905 \\
sulfomethoxazole & & & \\
\hline
\end{tabular}


isolation rates (\%) for $E$. coli in the non-CRF group and CRF group were $54.8 \%$ and $65.7 \%(\mathrm{p}=\mathrm{NS})$, and the isolation rates for K. pneumoniae were $12.2 \%$ and $13.9 \%$, respectively $(\mathrm{p}=\mathrm{NS})$. The isolation rates of other microorganisms did not differ between the two groups.

The antimicrobial sensitivity rates (\%) for E. coli according to DM are shown in Table 5. The antimicrobial sensitivities to ampicillin in the non-DM group and DM group were $30.6 \%$ and $35.7 \%$, respectively $(\mathrm{p}=$ 0.538 ), and sensitivities to gentamycin in the non-DM group and DM group were $67.2 \%$ and $63.4 \%(\mathrm{p}=0.656)$, respectively. The antimicrobial sensitivities to other antibiotics did not differ between the two groups.

\section{Discussion and Conclusions}

In this study, we attempted to determine whether there are differences between CRF and non-CRF patients in the bacteriologic patterns of community-acquired APN or in the antibiotic sensitivity patterns of uropathogens. This study showed that CRF does not seem to influence the isolation rates of different uropathogens or their susceptibility patterns to antimicrobials in patients with communityacquired APN that presented as a positive urine culture.

In general, CRF patients are known to be vulnerable to infection due to weakened immunity [6]. But little information is available regarding the role of CRF as a risk factor for the development of antimicrobial resistance in uropathogens. It has been reported that DM per se does not seem to influence the isolation rates of different uropathogens or their susceptibility patterns to antimicrobials [5]. In this study, the antimicrobial susceptibilities of microorganisms isolated in cases of community-acquired APN in non-DM and DM groups did not differ. However, the role of CRF in the etiology and antimicrobial resistance of uropathogens in patients with community-acquired APN has not been clarified.

It is known that $E$. coli is isolated in approximately $90 \%$ of APN cases [15]. Other studies have found that urinary Klebsiella is more frequent in diabetic patients compared to the detection rate in non-diabetic patients $[16,17]$. In this study, the most common cause of APN was E. coli (58.3\%, 293 cases), followed by K. pneumoniae (12.7\%, 64

Table 4 Isolation rate (\%) of uropathogens in APN patients with or without CKD

\begin{tabular}{llll}
\hline Uropathogens & Non-CKD & CKD & p-value \\
\hline E. coli & 54.8 & 65.7 & NS \\
K. pneumoniae & 12.2 & 13.9 & NS \\
Proteus & 2.7 & 3.0 & NS \\
Pseudomonas spp & 5.1 & 2.4 & NS \\
Enterococcus spp & 3.0 & 3.0 & NS \\
Other gram positive & 15.2 & 9.0 & NS \\
Other gram negative & 7.1 & 3.1 & NS \\
\hline
\end{tabular}

Table 5 Comparison between diabetes mellitus and nondiabetes mellitus patients with respect to antimicrobial sensitivity rate (\%) for $E$. coli

\begin{tabular}{llll}
\hline Antibiotics & Non-DM & DM & p-value \\
\hline Ampicilline & 30.6 & 35.7 & 0.538 \\
Cephalothin & 36.7 & 52.9 & 0.227 \\
Cefuroxime & 72.2 & 78.4 & 0.456 \\
Cefotaxime & 78.2 & 84.2 & 0.420 \\
Cefozolin & 55.3 & 81.8 & 0.106 \\
Ceftazidime & 80.0 & 80.6 & 0.942 \\
Cepefime & 81.4 & 84.2 & 0.690 \\
Imipenem & 95.7 & 100 & 0.195 \\
Gentamycin & 67.2 & 63.4 & 0.656 \\
Amikacin & 92.2 & 100 & 0.081 \\
Tobramycin & 84.5 & 86.5 & 0.767 \\
Ciprofloxacin & 71.2 & 62.6 & 0.674 \\
Trimethoprime-sulfomethoxazole & 56.8 & 61.0 & 0.638 \\
\hline
\end{tabular}

cases). Due to the inclusion of diabetic patients, K. pneumoniae was more common than in previous reports on APN. Among the microorganisms associated with APN, E. coli was found in $54.8 \%$ and $65.7 \%$ of cases in the non-CRF group and CRF group, respectively ( $\mathrm{p}=\mathrm{NS}$ ), and $\mathrm{K}$. pneumonia was present in $12.2 \%$ and $13.9 \%$ of cases, respectively $(\mathrm{p}=\mathrm{NS})$. There were no differences between the groups regarding the rates of other APN microorganisms.

In a previous analysis of the antibiotic sensitivity of E. coli in APN, the sensitivities to the first- and thirdgeneration cephalosporins, aminoglycoside and ciprofloxacin were greater than $90 \%$; $47 \%$ to ampicillin; and $60 \%$ to TMP-SMX [18]. In our current study, the rate of $E$. coli sensitivity was $96.9 \%$ to imipenem, $99.2 \%$ to amikacin, $90.8 \%$ to tobramycin, greater than $80 \%$ to third- and fourth-generation cephalosporin antibiotics, $71.7 \%$ to ciprofloxacin, and $61.4 \%$ to TMP-SMX. However, the sensitivity rates to gentamycin and ampicillin were low ( $42.9 \%$ and $39.4 \%$, respectively). Little data is available on the role of CRF as a risk factor for the development of antimicrobial resistance in uropathogens. Antimicrobial sensitivities to ampicillin in the non-CRF group and CRF group were $37.8 \%$ and $20.0 \%$, respectively $(\mathrm{p}=0.024)$, and those to gentamycin in the non-CRF group and CRF group were $71.9 \%$ and $51.9 \%$, respectively $(p=0.016)$. The antimicrobial sensitivities to other antibiotics did not differ between the two groups. In North America, a cut-off point of $20 \%$ has been suggested as the level of resistance at which an agent should no longer be used [19]. The observed high rates of $E$. coli resistance to ampicillin, cephalothin, and gentamycin precludes, at least in our area, the choice of these or similar drugs in the empirical initial treatment of community-acquired APN in CRF and non-CRF patients. 
In a recent case-control study, recent hospitalization and fluoroquinolone use in the previous six months were independent risk factors for fluoroquinolone resistance in community-onset febrile E. coli UTI [20]. In our study, we excluded cases of previous administration of antibiotics and hospital-acquired APN.

Recent cohort studies have suggested that CRF is also a risk factor for non-cardiovascular morbidity [21] and mortality, including those caused by infection $[22,23]$. Few studies have investigated the associations between CRF and specific infectious conditions. In this study, the value of HSCRP was $7.0( \pm 7.8) \mathrm{mg} / \mathrm{dL}$ in the non-CRF group and 11.0 $( \pm 9.4) \mathrm{mg} / \mathrm{dL}$ in the CRF group $(\mathrm{p}=0.002)$. The values of WBC $(\mathrm{p}=0.001)$ and ESR $(\mathrm{p}=0.03)$ were higher in the CRF group than they were in the non-CRF group. Based on these results, we propose that patients with CRF had higher values of inflammatory markers when they had APN and that more attention is needed in this area because community-acquired APN in CRF patients can be a serious illness.

The limitations of our study include performance on non-CRF and CRF patients admitted to a single hospital, although the study included a large number of patients. Second, the CRF group was older in age than was the non-CRF group. Finally, the results for blood culture were not included in this study.

Based on our results, ampicillin, cephalothin, and gentamycin should not be considered as an initial therapeutic regimen in either CRF or non-CRF patients with community-acquired APN. In our series of patients with community-acquired APN presenting with a positive urine culture test, CRF per se does not seem to influence the isolation rates of different uropathogens or their susceptibility patterns to antimicrobials. A detailed prospective study is required to address the influences of CRF on the spectrum and antimicrobial susceptibility of the uropathogens involved in community-acquired APN.

\section{Authors' contributions \\ YSJ, M.D. and HSS, M.D. participated in the design of the study and performed the statistical analysis. HR, M.D. conceived of the study, and participated in its design and coordination. All authors read and approved the final manuscript.}

\section{Competing interests}

This study was supported by a grant from the Kosin University College of Medicine (2010).

Received: 27 December 2010 Accepted: 21 April 2011

Published: 21 April 2011

\section{References}

1. Stamm WE, Hooton TM: Management of urinary tract infections in adults. N Engl J Med 1993, 329:1328-34.

2. Hooton TM, Stamm WE: Diagnosis and treatment of uncomplicated urinary tract infection. Infect Dis Clin North Am 1997, 11:551-81.

3. Gupta K, Hooton TM, Wobbe CL, Stamm WE: The prevalence of antimicrobial resistance among uropathogens causing acute uncomplicated cystitis in young women. Int J Antimicrob Agents 1999, 11:305-8.

4. Scholes D, Hooton TM, Roberts PL, Gupta K, Stapleton AE, Stamm WE: Risk factors associated with acute pyelonephritis in healthy women. Ann Intern Med 2005, 142:20-7.

5. Bonadio M, Costarelli S, Morelli G, Tartaglia T: The influence of diabetes mellitus on the spectrum of uropathogens and the antimicrobial resistance in elderly adult patients with urinary tract infection. BMC Infect Dis 2006, 6:54.

6. Descamps-Latscha B, Chatenoud L: T cells and b cells in chronic renal failure. Semin Nephrol 1996, 16:183-91.

7. Bergman SJ, Speil C, Short M, Koirala J: Pharmacokinetic and pharmacodynamic aspects of antibiotic use in high-risk populations. Infect Dis Clin North Am 2007, 21:821-46.

8. Safrin S, Siegel D, Black D: Pyelonephritis in adult women: Inpatient versus outpatient therapy. Am J Med 1988, 85:793-8.

9. Garner JS, Jarvis WR, Emori TG, Horan TC, Hughes JM: CDC definitions for nosocomial infections, 1988. Am J Infect Control 1988, 16:128-40.

10. Report of the expert committee on the diagnosis and classification of diabetes mellitus. Diabetes Care 2003, 26(1):S5-20.

11. Stevens LA, Coresh J, Greene T, Levey AS: Assessing kidney functionmeasured and estimated glomerular filtration rate. N Engl J Med 2006, 354:2473-83.

12. Johnson CA, Levey AS, Coresh J, Levin A, Lau J, Eknoyan G: Clinical practice guidelines for chronic kidney disease in adults: Part i. Definition, disease stages, evaluation, treatment, and risk factors. Am Fam Physician 2004, 70:869-76.

13. Jorgensen $\mathrm{JH}$, Hindler JF: New consensus guidelines from the clinical and laboratory standards institute for antimicrobial susceptibility testing of infrequently isolated or fastidious bacteria. Clin Infect Dis 2007, 44:280-6.

14. Livermore DM: Beta-lactamases in laboratory and clinical resistance. Clin Microbiol Rev 1995, 8:557-84.

15. Nicolle LE: Uncomplicated urinary tract infection in adults including uncomplicated pyelonephritis. Urol Clin North Am 2008, 35:1-12.

16. Bonadio M, Meini M, Spitaleri P, Gigli C: Current microbiological and clinical aspects of urinary tract infections. Eur Urol 2001, 40:439-44, discussion 445.

17. Stapleton A: Urinary tract infections in patients with diabetes. Am J Med 2002, 113(1):80S-4S.

18. Bean DC, Krahe D, Wareham DW: Antimicrobial resistance in community and nosocomial escherichia coli urinary tract isolates, Iondon 2005-2006. Ann Clin Microbiol Antimicrob 2008, 7:13.

19. Gupta K, Hooton TM, Stamm WE: Increasing antimicrobial resistance and the management of uncomplicated community-acquired urinary tract infections. Ann Intern Med 2001, 135:41-50.

20. van der Starre WE, van Nieuwkoop C, Paltansing S, Van't Wout JW, Groeneveld GH, Becker MJ, Koster T, Wattel-Louis GH, Delfos NM, Ablij HC, Leyten EM, Blom JW, van Dissel JT: Risk factors for fluoroquinoloneresistant escherichia coli in adults with community-onset febrile urinary tract infection. J Antimicrob Chemother 2010, 1-7.

21. Tonelli M, Wiebe N, Culleton B, House A, Rabbat C, Fok M, McAlister F, Garg AX: Chronic kidney disease and mortality risk: A systematic review. J Am Soc Nephrol 2006, 17:2034-47.

22. Nissenson AR, Collins AJ, Hurley J, Petersen H, Pereira BJ, Steinberg EP. Opportunities for improving the care of patients with chronic renal insufficiency: Current practice patterns. J Am Soc Nephrol 2001, 12:1713-20.

23. Fried LF, Katz R, Sarnak MJ, Shlipak MG, Chaves PH, Jenny NS, StehmanBreen C, Gillen D, Bleyer AJ, Hirsch C, Siscovick D, Newman AB: Kidney function as a predictor of noncardiovascular mortality. J Am Soc Nephrol 2005, 16:3728-35.

Pre-publication history

The pre-publication history for this paper can be accessed here: http://www.biomedcentral.com/1471-2334/11/102/prepub

doi:10.1186/1471-2334-11-102

Cite this article as: Jung et al:: The influence of chronic renal failure on the spectrum and antimicrobial susceptibility of uropathogens in community-acquired acute pyelonephritis presenting as a positive urine culture. BMC Infectious Diseases 2011 11:102. 